\title{
Filtering of NDT signals obtained from wrapped steel cables
}

\author{
R. Christen, A. Novakovic, A. Bergamini and M. Motavalli \\ Empa, Swiss Federal Laboratories for Material Testing and Research \\ Ueberlandstrasse 129, CH-8600 Duebendorf, Switzerland;
}

\begin{abstract}
The main cables of suspension bridges are often wrapped with a steel wire, in order to compact the cable and hold it in shape. If a non-destructive evaluation by means of magnetic methods is performed on such a cable, disturbances due to the wrapping can be expected in the measured signal. In the presented work, these disturbances shall be quantified and compared to the flaw signals. Different approaches for the separation of the disturbance and the flaw signal are discussed. Additionally, the possibility to detect wire breaks and corrosion within an unwrapped steel cross-section could be shown in laboratory measurements. The influence of the wrapping was investigated using finite element (FE) simulations and experimental laboratory measurements. A parameter study was performed in order to obtain data in which the components from a flaw and the wrapping can be separated. The parameters varied in this study were chosen depending on the prospect of success and the cost of the realization. Using these data sets different filtering methods, such as wavelet analysis, were implemented. A final comparison of the different methods suggests the most efficient way to assess the condition of such cable systems using magneto-inductive testing. Finally, it can be concluded that the use of FE simulation is a very useful tool for the development of new data analysis methods, even if a real set-up and data from measurements exist.
\end{abstract}

Keywords: NDE, magneto-inductive, signal processing, suspension bridges

\section{INTRODUCTION}

In the past years, a system for the inspection of steel cables with large diameters $(\varnothing>130 \mathrm{~mm})$ was developed at Empa. In the summer of 2001 this system was used to inspect the stay cables of the RAMA IX bridge in Bangkok, Thailand. ${ }^{1}$ While the mechanical part of the measurement system was not developed further, some new methods for the data analysis were implemented in the meantime. ${ }^{2,3}$ In order to gain access to an additional market in the field non-destructive testing of civil engineering structures thoughts on the use of this measuring system on the main cables of suspension bridges were made. The main differences to a stay cable are the larger diameter of the main cables and the presence of vertical hangers, which are connected to the bridge deck and divide the main cable into relatively short sections of a few meters. The needed energy to magnetize such a cable with a diameter of several hundred millimeters increases dramatically and hence the design of the measuring system has to be revised. Due to the short distance between the hangers, the installation of the measuring device on the cable has to be as simple as possible, in order to enable an efficient and economical testing. Additionally, these cables are often wrapped with a steel wire for different purposes. Between each of these windings a gap is formed which is almost perpendicular to the cable main axis. Each gap represents a finite surface slot which will generate a magnetic flux leakage (MFL) signal that is detected by the measuring device. Consequently, there are two main difficulties to be overcome:

- The magnetization of very large cables and the handling of the device.

- Filtering of the disturbances caused by the steel wrapping.

Before a new measuring device is designed to address the problem of the magnetization, the disturbance in the measured signal shall be investigated using finite element $(\mathrm{FE})$ calculations and laboratory measurements. In the field of nondestructive inspection of pipelines and steel cables FE models are often used for the characterization or localization of flaws. $^{4,5}$ In this work, the FE model will be used to perform a parameter study, while the selection of the parameters to be varied is discussed in detail. Based on these calculations, different analysis and filtering techniques will be applied to the data.

Further author information: E-mail: rouven.christen@empa.ch, Telephone: +41 448234530

Nondestructive Characterization for Composite Materials, Aerospace Engineering,

Civil Infrastructure, and Homeland Security 2007, edited by H. Felix Wu, Aaron A. Diaz, Peter J. Shull

Proc. of SPIE Vol. 6531, 653110, (2007) $\cdot 0277-786 \times / 07 / \$ 18 \cdot$ doi: $10.1117 / 12.715119$

Proc. of SPIE Vol. 6531 653110-1 


\section{EXPERIMENTAL SET-UP}

The experimental set-up, which was installed in Empa's testing laboratory, consists of a parallel wire bundle and two passive anchorages. The outer diameter of the cable is approximately $125 \mathrm{~mm}$ and the free length is about $3.5 \mathrm{~m}$, while the anchorage region is $1.5 \mathrm{~m}$ on each side. The whole bundle consists of approximately 200 wires with a diameter of $7 \mathrm{~mm}$. Artificial flaws were introduced to simulate different damage scenarios. Among others, a $1.2 \mathrm{~m}$ long bundle of 14 corroded steel wires was introduced in the cable (see figure 1). For this purpose some wires in the cable were cut and replaced by the corroded bundle.
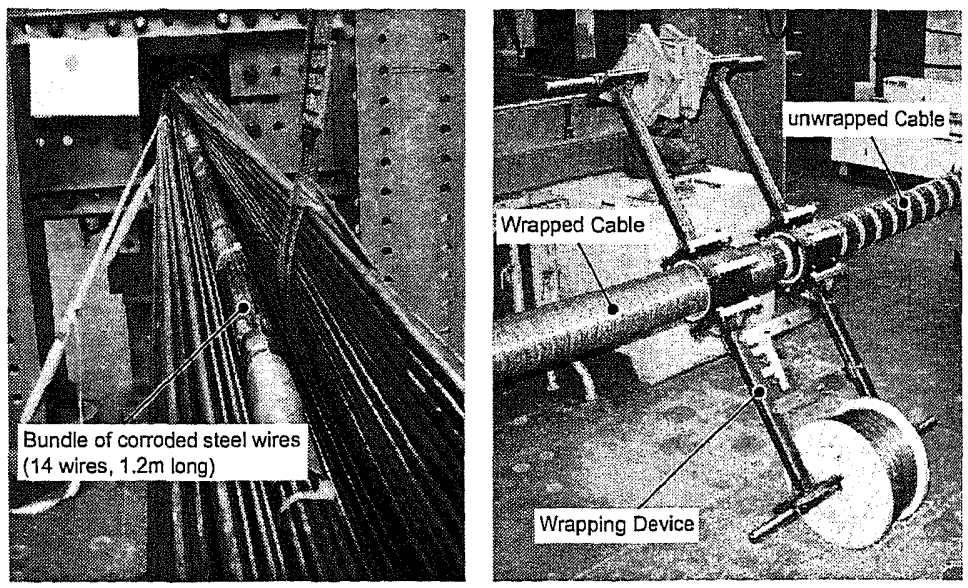

Figure 1. Test set-up installed in Empa testing laboratory during the installation of the corroded steel wires (left) and the wrapping procedure (right).

The used NDT device is based on an electromagnet, which is used to generate the necessary magnetic fields to saturate the whole steel cross-section. A coil with approximately 150 turns is wounded around the cable, while the body of the coil consists of several individual elements. These elements can be arranged in different configuration to fit cable diameters from $100 \mathrm{~mm}$ to $250 \mathrm{~mm}$. To magnetize the cable, a current up to $100 \mathrm{~A}$ is circulated in the coil, which produces magnetic fields in the range of $50 \mathrm{kA} / \mathrm{m}$. Additionally, each element includes two sensing coils to measure the magnetic leakage field. The configuration for the cable used in this work consists of 19 sensors and has a diameter of $135 \mathrm{~mm}$ and consequently a sensor lift-off of about $5 \mathrm{~mm}$.
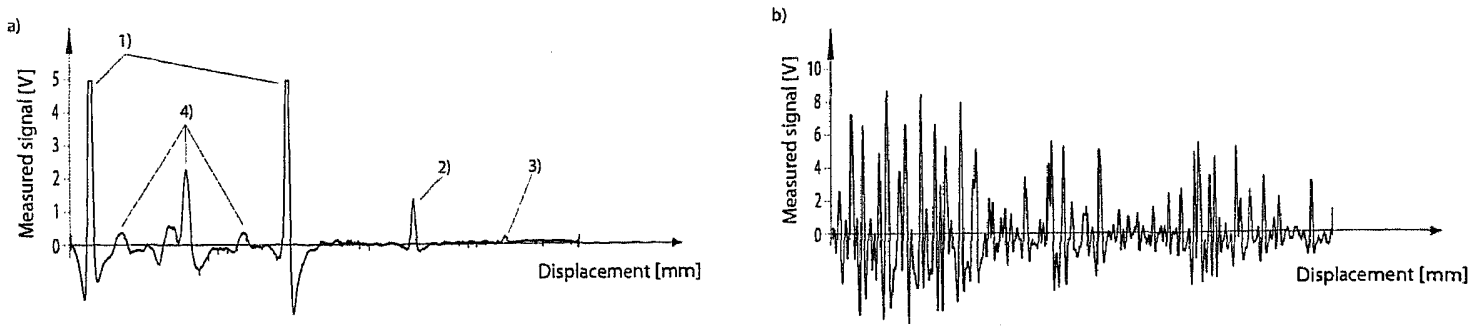

Figure 2. Measured data of the unwrapped (a) and wrapped (b) cable.

Figure 2a shows the signal measured on the test set-up in the laboratory. The different flaw signals can be recognized in the unwrapped signal (compare to table 1). In the second measurement the original signal is covered by many additional features caused by the wrapping.

The signal within the corroded part show several changes in the magnetic flux leakage field (flaw number 4), originated from changes in the cross-sectional area due to corrosion. The signal from the artificial flaw number 3 (saw cut with $0.25 \%$ reduction of the steel cross-section) is actually too small to be identified without the knowledge of its position. The measured signal is a voltage, which is induced in the pick up coils by the change of the radial component of the flux leakage 
field. The measured voltage can be described as:

$$
U=A_{v} \cdot N \cdot \frac{d\left(\int_{A} \vec{B} \cdot d \vec{A}\right)}{d t}
$$

where $A_{v}$ is the gain of the pre-amplifiers, $N$ the number of turns of the pick-up coils, $\vec{B}$ the flux leakage field and $\vec{A}$ the area of the pick-up coils. When the sensor area is parallel to the cable surface and the velocity $v=d z / d t$ of the measuring device is constant, the measured signal can be expressed as:

$$
U=A_{v} \cdot N \cdot \frac{d B_{r}}{d z} \cdot v \cdot A
$$

where $B_{r}$ is the radial component of the magnetic flux leakage field and $A$ is the absolute value of the area vector $\vec{A}$.

Table 1. Summary of the artificial flaws introduced in the laboratory cable model.

\begin{tabular}{lllll}
\hline Number & Flaw type & Length of flaw & Number of wires & Loss in steel cross-section \\
\hline 1$)$ & Saw cut & few millimeter & 14 & appr. $7 \%$ \\
$2)$ & Saw cut & few millimeter & 1 & appr. 0.5\% \\
$3)$ & Saw cut & few millimeter & 0.5 & appr. 0.25\% \\
$4)$ & Corrosion & within 1.2m & N.A. & N.A. \\
\hline
\end{tabular}

\section{FINITE ELEMENT MODEL}

\subsection{Structure of the model}

For modeling purposes a finite element program called MagNet $v 6.0$ from Infolytica Corp. was used. The parallel wire bundle was modeled as a $2 \mathrm{~m}$ long solid body with the same steel cross sectional area as the real cable. The magnetic material properties of the cable were estimated based on a measured B-H curve of a common strand used for stay cable systems. The applied magnetic field $H_{a}$ was chosen as $45 \mathrm{kA} / \mathrm{m}$, which is comparable to the value in the real measurement. The system was modeled in 2D in order to minimize the calculation time. The magnetic flux was calculated along a line parallel to the surface of the body at a distance similar to the lift off the sensors in the measurement have. If a cylindrical object, such as a steel cable, is wrapped with steel wire, the gap between two turns is almost perpendicular to the cable main axis and hence to the direction of the magnetization. Therefore each gap represents a discontinuity producing a magnetic flux leakage field. For this reason the wrapping had to be modeled by means of additional elements.

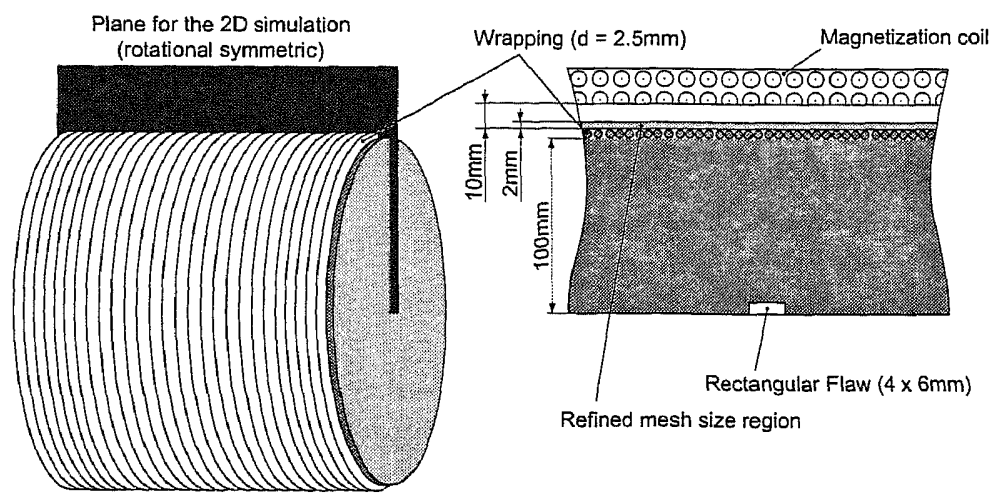

Figure 3. On the left a 3D view of a wrapped steel cable is shown with the 2D plane used in the simulation. The right side shows a part of the finite element model with randomly distributed wire objects. 
As seen in figure 3 circular steel elements with a diameter of $2.5 \mathrm{~mm}$ each were introduced into the model to represent the wrapping ( 800 objects over the whole model length). For the first calculations the gap between the elements was chosen to be zero, representing a perfectly tight wrapping. In later simulations the gap width between two elements was increased and varied to investigate its influence on the calculated magnetic flux leakage field. Therefore, two further calculations with a constant gap width of $1.25 \mathrm{~mm}$ and a randomly generated gap width pattern, respectively were performed. Table 2 shows a summary of the gap widths used for the three FE models, while the position of small, medium and large gaps along the cable was determined randomly in simulation three. Additionally, a rectangular flaw $(4 \mathrm{~mm} \times 6 \mathrm{~mm})$ was introduced in

Table 2. Different gap sizes between the wrapping elements used in the three FE models.

\begin{tabular}{cccc}
\hline Model No. & Gap type & Gap size & Percentage \\
\hline 1 & No gap $(N G)$ & $0 \mathrm{~mm}$ & $100 \%$ \\
\hline 2 & Large equal gaps $(L E G)$ & $1.25 \mathrm{~mm}$ & $100 \%$ \\
\hline 3 & Small gaps $(S G)$ & $0-0.175 \mathrm{~mm}$ & $97.5 \%$ \\
3 & Medium gaps $(M G)$ & $0.5125-0.6875 \mathrm{~mm}$ & $1.5 \%$ \\
3 & Large gaps $(L G)$ & $1.1125-1.2875 \mathrm{~mm}$ & $1 \%$ \\
\hline
\end{tabular}

the center of the model. In order to obtain an accurate result, a fine solution mesh was needed. For this purpose, a maximal mesh size of a few millimeter was defined for all objects. Difficulties arise, when the optimal mesh size for the air box, which surrounds the whole model, has to be found. On one hand, the air box has to be large enough (several times the size of the entire model) to avoid an influence on the calculation results. On the other hand, the mesh on the surface of the cable has to be very fine, because the magnetic flux leakage field at this position is of interest. If a refined mesh is defined for the whole air box, the maximum number of elements would be exceeded. Therefore, a small air box with a height of $2 \mathrm{~mm}$ was introduced with a maximum mesh size of $0.5 \mathrm{~mm}$, while the mesh size within the rest of the air box was not limited.

\subsection{Parameter study}

The objective of the presented work is to separate the two components of the signal generated by the wrapping and the flaw, respectively. This goal can be reached by applying a suitable filtering method, or an other postprocessing algorithm. As an alternative to this, the measurement technique, or one of its parameters may be adapted in a certain way to obtain a second signal from the same object. If the changed parameter affects the two components in the signal in a different manner, the two signals may be used to separate the disturbance from the flaw signal. Because the change of any parameter of an existing measurement device is connected to large expenses, the creation of a FE model of the given problem was considered to be very useful.

In order to perform a parameter study, we have to clarify which parameters can be varied. In some cases the amount of variable parameters is too large, which makes a well structured parameter study almost impossible. For this reason, the second question is, which variable parameters shall be used for the study and why. In the case of MFL measurements the number of possible parameters is not very large.

1. Intensity of the magnetization current

2. Number of turns in the magnetization coil

3. Lift-off of the sensors

4. Frequency of the magnetization current

Obviously, parameter (1) and (2) are equivalent, because in fact both change the level of the magnetic field in the sample. Therefore we end up with three parameters which can be changed. Although this is a manageable number of parameters, we wanted to estimate the probability of success and the measures that have to be taken in order to realize the variation of each parameter in the real application. The following list shows a summary of the expected results. 


\section{Magnetic field level:}

If the magnetic field strength is reduced the signal amplitude will decrease. As we know the flaws in the center of a cable cannot be detected if the sample is not saturated magnetically, while the signal of the disturbance is measured with a lower amplitude. Therefore it is assumed, that the different influence on the two components of the signal may allow for a separation of them.

Moreover, this parameter can be changed easily if the measuring device is based on electro magnets as in this example.

\section{Sensor lift-off:}

The change of the sensor lift-off, which is defined as the distance between the cable surface and the sensor, has an influence on the measured signal. For a single crack or subsurface flaw the signal amplitude and width changes, but not the general signal shape. Because this is the case for all types of discontinues, this parameter is not assumed to give useful results in terms of the separation of the signal components. Even though, the effect of the superposition of many cracks, which are located close to each other may be of interest.

Because the body of the coil is built from individual segments, the realization of a changing sensor lift-off is possible within a given limit. An additional problem in the real case application might be the surface of the cable, which has to be very clean and smooth to perform measurements with a low sensor lift-off.

\section{Frequency of the magnetization current:}

By increasing the frequency of the magnetization current, the distribution of the magnetic flux within the cable crosssection will be influenced by additional phenomena like the skin effect. The basic idea behind the change of this parameter is similar to the first one. The disturbance shall be measured without getting any information about the subsurface flaw, in order to separate the disturbance from the flaw signal.

High frequency measurements are difficult to realize due to the relatively high impedance of the magnetization coil. Moreover the changing background field will produce a signal in the sensing coils.

On a first view, parameter one has the highest prospect of success, because it is expected to be possible to measure the pure disturbance. Nevertheless, the second parameter is included in the parameter study as well, because the consequences of an increase of the sensor lift-off are difficult to estimate, while the expense for the realization is comparably low. Thus the third parameter is the only one, which was not used in the parameter study. The values for the current and lift-off variation are summarized in table 3 .

Table 3. Summary of the performed calculations with corresponding values used for the two parameters.

\begin{tabular}{ccc}
\hline Simulation ID & Magnetization current & Sensor lift-off \\
\hline C1 & $20 \mathrm{~A}$ & $1 \mathrm{~mm}$ \\
$\mathrm{C} 2$ & $40 \mathrm{~A}$ & $1 \mathrm{~mm}$ \\
$\mathrm{C} 3$ & $60 \mathrm{~A}$ & $1 \mathrm{~mm}$ \\
$\mathrm{C} 4$ & $80 \mathrm{~A}$ & $1 \mathrm{~mm}$ \\
$\mathrm{C} 5$ & $80 \mathrm{~A}$ & $6.5 \mathrm{~mm}$ \\
\hline
\end{tabular}




\section{SIMULATION RESULTS}

The results from the calculation with a varying magnetization current confirmed the assumption, that the flaw cannot be detected with a low magnetization, while the signal of the wrapping is scaled. To quantify this scaling, the root mean square (RMS) value, which represents the signal energy $S$, is calculated for each signal and plotted in figure 4a with respect to the magnetization current $I$.
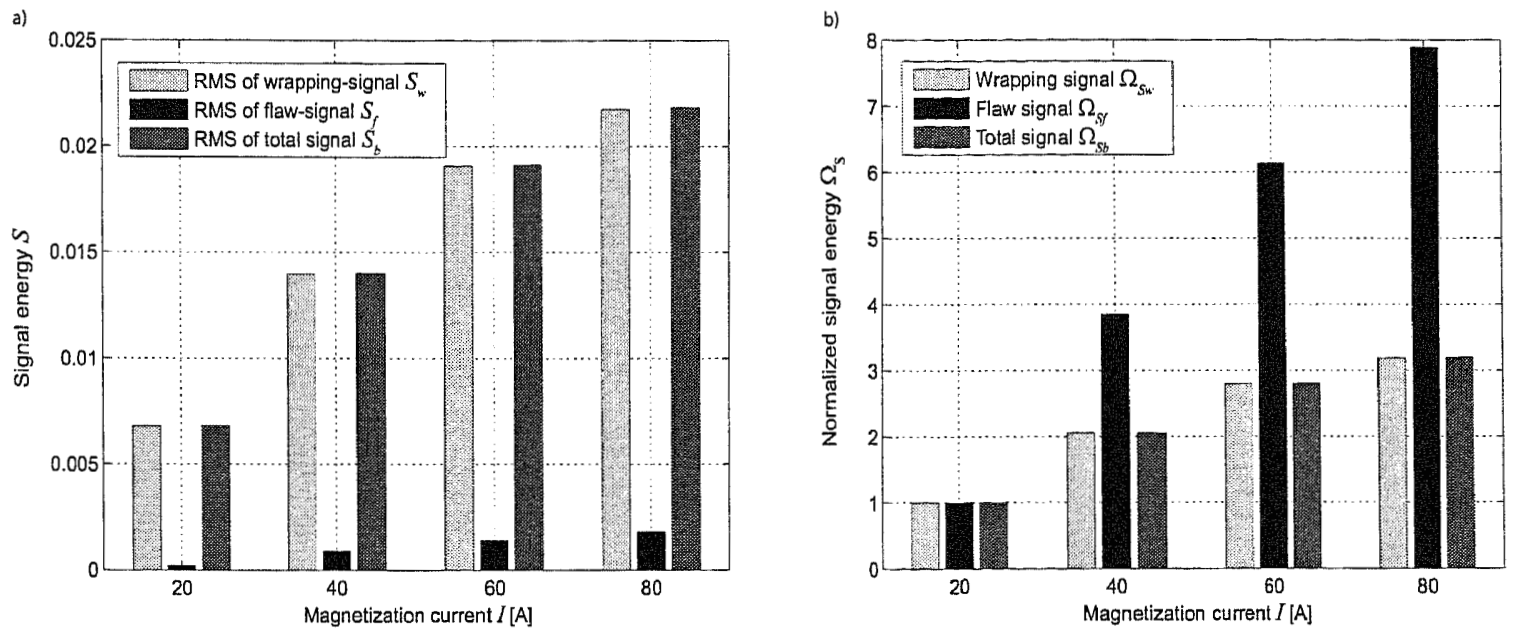

Figure 4. a) Comparison of the signal energy $S$ of wrapping, flaw and total signal. b) Normalized energies $\Omega_{S}$ make clear the energy increase for the different signals.

The nonlinear behavior of the signal energies is caused by the nonlinear magnetic properties of the steel. Once the sample is magnetically saturated, the amplitude of the measured leakage field does not increase anymore. In figure $4 \mathrm{~b}$ the values are normalized, so that:

$$
\Omega_{S}=\frac{S(I)}{S\left(I_{0}\right)}
$$

where $S\left(I_{0}\right)$ is the measurement at the lowest magnetization level, which is $20 \mathrm{~A}$ in this case. It can be seen, the the energy in the flaw signal increases by a factor $F_{f} \approx 8$ when the the current is increased from $20 \mathrm{~A}$ to $80 \mathrm{~A}$. However, the wrapping signal and the total signal, which represents the normalized energy of a signal with both components, increase only by a factor $F_{w} \approx F_{b} \approx 3$. The scaling factor $F_{b}$ is almost the same as $F_{w}$, because the additional energy introduced by the flaw is very small compared to the total energy of the wrapping signal. Based on these results, it seems to be possible to separate the two components in the signal by applying the following equation:

$$
r=s_{b}(x, 80 A)-F_{b} \cdot s_{b}(x, 20 A)
$$

Where $s_{b}(x, I)$ is the total signal containing both components, calculated for a specific magnetization current $I$. In the resulting signal $r$ the disturbance and a part of the flaw signal are removed. Due to the much higher increase in the flaw signal energy, a considerable portion of the signal remains in $r$.

The phase shift $\varphi$ between the two signals is very important for this kind of evaluation, because already a small variation makes it impossible to apply this method. Nevertheless, it should be investigated, if this approach can be applied at least to simulated data, because the accuracy of the phase is rather a problem in the real measurement application. If equation 4 is applied to the simulated data, the results are not very promising, although the phase $\varphi$ is exactly zero. A possible reason for the bad results is the accuracy of the scaling factor $F_{b}$ which is calculated over the whole signal length and therefore is an average scaling factor. The following equation is true for an position invariant scaling factor:

$$
\sum\left(\left(s_{w}(x, 80 A)-F_{b} \cdot s_{w}(x, 20 A)\right)^{2}\right)=0
$$


Indeed equation 5 is $\neq 0$ and therefore this approach can only be applied to an idealized system and neither in a FE simulation, nor in a real measurement the condition in equation 5 can be achieved. Finally it can be concluded, that this method is not suitable to eliminate the wrapping signal, although the statement made in section 3.2 could be confirmed by the FE calculations.

As mentioned above, we would expect a change in the signal width and amplitude, when the sensor lift-off is increased. In the case of a single flaw the signal amplitude is reduced and the peak broadens slightly when the lift-off is increased. This can be confirmed by the FE calculation, as well as by analytical models such as the Förster's approximation in equation 6 , which describes the magnetic flux leakage produced by a finite surface slot and a relatively large sensor lift-off $\left(\{x, y\}>L_{g}\right)$.

$$
H_{y}(x, y)=\frac{-H_{g} \cdot L_{g}}{\pi}\left(\frac{x}{x^{2}+y^{2}}-\frac{x}{x^{2}+\left(Y_{0}+y\right)^{2}}\right)
$$

Where, $L_{g}$ represents the crack width, $Y_{0}$ the crack depth and $y$ the lift-off of the sensor. $H_{g}$ includes various geometrical parameters of the magnetic circuit as well as the relation between the field strength within the gap and the applied magnetic field strength $\left(H_{a}\right) .{ }^{6}$ In figure 5 two calculations with different lift-off values are compared.
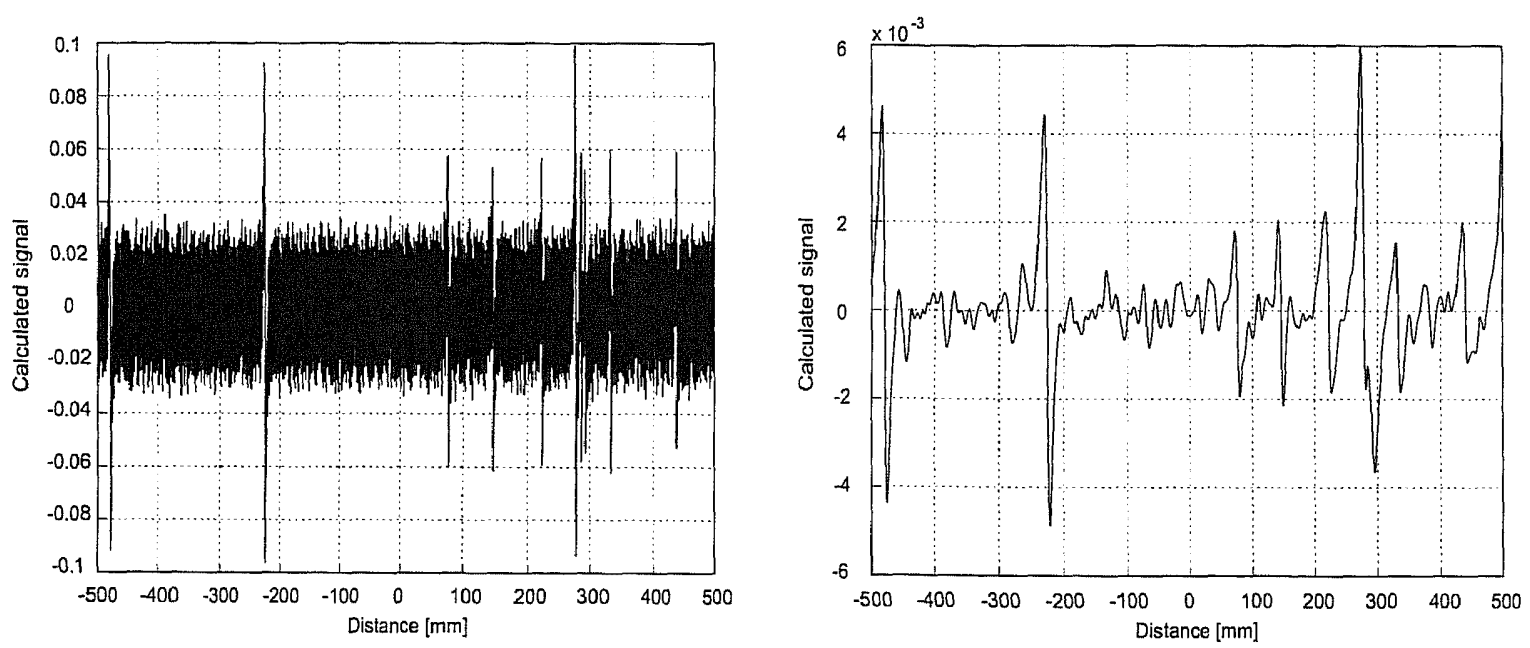

Figure 5. Left: Calculated signal originated by the wrapping with different gaps and a sensor lift-off of $1 \mathrm{~mm}$ (C4). Right: Same calculation with a sensor lift-off of $6.5 \mathrm{~mm}$ (C5).

Apparently, the high frequency components are removed form the signal, when the lift-off is increased. The peaks at the position of larger gaps broadened remarkably and the overall signal energy drops by a factor of twenty. It is assumed, that the higher frequency signals erase each other due to their broadening. Both signals show flaw-like features, although this simulation was made without including any flaw in the model. Therefore it is clear, that the filtering of this signals is a challenging task.

\section{FILTERING METHODS}

\subsection{Low-pass filtering}

Since the disturbance, as well as the useful signal generated by a flaw have the same physical origin, it can be expected, that the two signals have a high degree of similarity. Both are caused by local changes in the permeability, one within the cable cross-section and the other on the cable surface. As seen in section 4 the signal of the surface slot is considerably sharper than the signal from the subsurface flaw, at least for a small sensor lift-off. This can be explained by the small distance between the surface gap and the position of the sensor (where the $B_{y}$ field is calculated). This difference in the frequency should allow for the filtering of the signal using a simple low-pass filter. The main question is, how the superposition of the signals of several gaps looks like. In the graph a1) on the top left side of figure 6 the results of the simulation with tight wrapping (model number 1 ) is shown. If the signal is transformed to the frequency domain using the 
Fast Fourier Transformation (FFT), a large peak can be found at $0.4 \mathrm{~mm}^{-1}$, which is the reciprocal of the wire diameter of $2.5 \mathrm{~mm}$. Additionally, some weak peaks at higher harmonics can be found. From this spectrum it can be deduced that the superposition of several gaps leads to a slightly distorted sine like signal. Because the frequencies of the flaw signal are much lower, namely in the range of 0 to $0.05 \mathrm{~mm}^{-1}$, the disturbance can be eliminated using a butterworth low-pass filter, as shown in graph a3) of figure 6 . The sine like shape of the magnetic flux leakage field could be confirmed by performing another simulation with large equal gaps between the wires (simulation number 2). In the frequency spectrum of this signal the main peak is located at $0.26 \mathrm{~mm}^{-1}$, which is equal to $1 /(2.5 \mathrm{~mm}+1.25 \mathrm{~mm})$. In a real application the wrapping would never be as homogenous and tight as in these calculations. Therefore, the same calculation is made using the model with varying gap widths as described in the previous section. Due to the range of gap widths ( $0 \mathrm{~mm}$ to $1.2875 \mathrm{~mm}$ ), a spectrum with frequencies between $0.26 \mathrm{~mm}^{-1}$ and $0.4 \mathrm{~mm}^{-1}$ would be expected and the same low-pass filter should remove the disturbance.
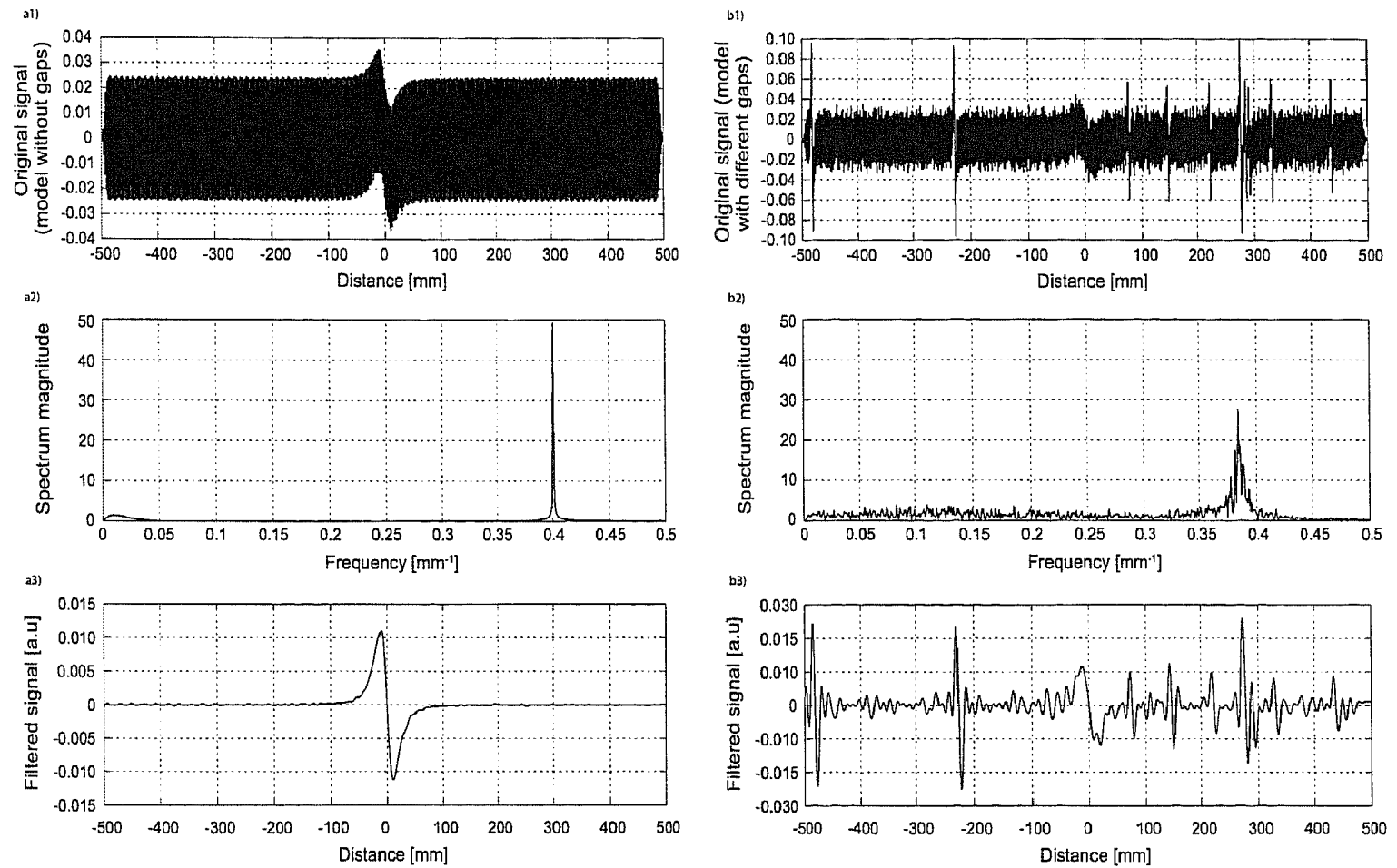

Figure 6. a1) Calculated magnetic flux leakage field $B_{y}(x)$ (model 1 tight wrapping), a2) spectrum of the signal with a strong peak at $0.4 \mathrm{~mm}^{-1}$, a3) filtered signal (butterworth low-pass $13^{\text {th }}$ order). b1) Calculated magnetic flux leakage field $B_{y}(x)$ (model 3 different gaps), b2) the main peak is slightly shifted toward a lower frequency due to the presence of some larger gaps. The frequency components of the flaw $\left(0\right.$ to $\left.0.05 \mathrm{~mm}^{-1}\right)$ are less clear than in example a2). b3) filtered signal (butterworth low-pass $13^{\text {th }}$ order).

As seen in figure 6 graph b3), the low-pass filter fails to filter out the disturbance and graph b2) shows that this signal includes some low frequency components in the same range as the flaw signal is. Consequently, these calculations show, that the signal has a sine like shape only, when the gaps between the wires are distributed evenly (equal gap widths).

\subsection{Wavelet de-noising}

In the previous section the influence of different gap widths and distributions were discussed. While the disturbance could be filtered out from the results of simulation number 1 and 2 , this was not possible for the last and most realistic case. The difference between the simulations with low and high sensor lift-off will be investigated in this section. A stationary wavelet decomposition of the signals shown in figure 5 will be used, in order to determine if the two signals have common features or not. To do so, a standard wavelet from the reverse biorthogonal wavelet family was used. The characteristic 
shape of this wavelet is similar to the shape of the radial magnetic leakage fiux we measure on the surface of a cable, hence it is assumed to be well suited to extract those features from a signal. Figure 7 shows the result of this decomposition and it can be seen, that the two signals are very different at the lower levels, while they are very similar at higher levels. To quantify the similarity, the cross correlation vector $C$ of each decomposition level is calculated. ${ }^{7}$ This vector has a

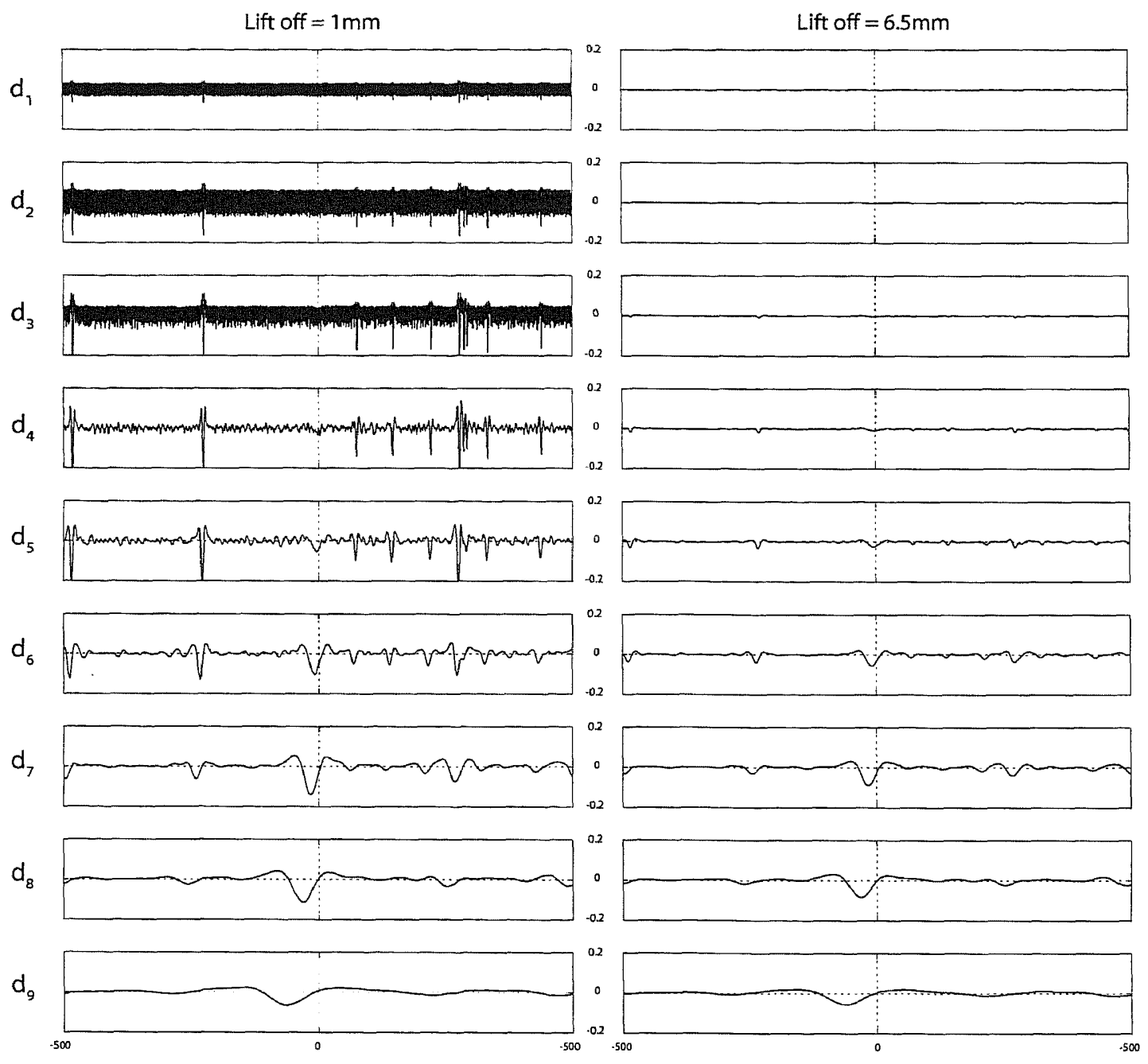

Figure 7. Stationary wavelet decomposition (level 1 to 9) for $1 \mathrm{~mm}$ (left) and $6.5 \mathrm{~mm}$ (right) sensor lift-off.

length of $2 \cdot N-1$, where $N$ is the length of the corresponding signal vectors. The position of the maximum value in vector $C$ shows the necessary phase between the two input signals to achieve an optimal correlation, while the value of this maximum is a measure for the quality of the correlation and thus the similarity itself. Each correlation vector has it's maximum value in the middle, or when the signals are in phase, respectively. In order to be able to compare the cross correlation value, the input vectors have to be normalized in a way, that the signal energy is equal to one. This is done by dividing each signal by its RMS value. The resulting cross correlation coefficient is then normalized with the signal length. This was done for the decomposition levels one to twelve and the results are summarized in table 4 . As it can be seen the value increases from almost zero towards one for the last few decomposition levels.

If a wavelet de-noising is performed, usually a decomposition is made and the levels which include the components to be removed are set to zero before an inverse decomposition is performed. Indeed, this is what happens when the lift-off is increased from $1 \mathrm{~mm}$ to $6.5 \mathrm{~mm}$ in the FE simulation. When a wavelet de-noising on the signal obtained from calculation $\mathrm{C} 4$ is performed by removing the components in level $d_{1}$ to $d_{5}$, we would generate almost the same pattern as we obtain 
Table 4. Maximal values of the normalized cross correlation vectors for all signals (decomposition level one to twelve).

\begin{tabular}{ccccccc}
\hline Decomposition level & $\mathrm{d} 1$ & $\mathrm{~d} 2$ & $\mathrm{~d} 3$ & $\mathrm{~d} 4$ & $\mathrm{~d} 5$ & $\mathrm{~d} 6$ \\
Normalized correlation value & 0.0604 & 0.0728 & 0.2146 & 0.5360 & 0.7484 & 0.9153 \\
\hline Decomposition level & $\mathrm{d} 7$ & $\mathrm{~d} 8$ & $\mathrm{~d} 9$ & $\mathrm{~d} 10$ & $\mathrm{~d} 11$ & $\mathrm{~d} 12$ \\
Normalized correlation value & 0.9806 & 0.9934 & 0.9980 & 0.9980 & 0.9997 & 0.9997 \\
\hline
\end{tabular}

from calculation C5. If we try to do a wavelet de-noising on signal C5, either nothing or all information, including the flaw, is removed. This is because all levels from $d_{6}$ to $d_{9}$ show signal components from the flaw and the wrapping as well. Finally, it can be summarized, that it does not appear possible to remove the disturbance in the signal by using the wavelet de-noising technique.

\subsection{Signal synthesis}

In contrast to an application where the disturbance is originated by any unknown physical process, the origin of the disturbance in this example is given by the position of the wires. Under the precondition that we know the exact position of each wire, which is the case for the data obtained from the FE calculation, it should be possible to perform a synthesis of the wrapping signal. A simple subtraction of the signals would lead to the flaw signal. Some practical problems, such as the determination of the wrapping positions and the correct phase between the two signals, have to be solved before it can be applied in the real application. Nevertheless, the success of this method should be investigated theoretically using the FE simulations.

To generate the model, a script was used to place the wire objects on the surface of the cable and therefore a vector containing all gaps and consequently all wire positions is available. Using equation 6 , a signal was calculated for each gap, while the function $H_{y}(x)$ was shifted to the position of the gap. The gap width was used to adapt the parameters $y$ and $Y_{0}$, hence changing the signal shape. By adding all these signals, a signal pattern could be generated which is similar to the one obtained from the FE calculation.

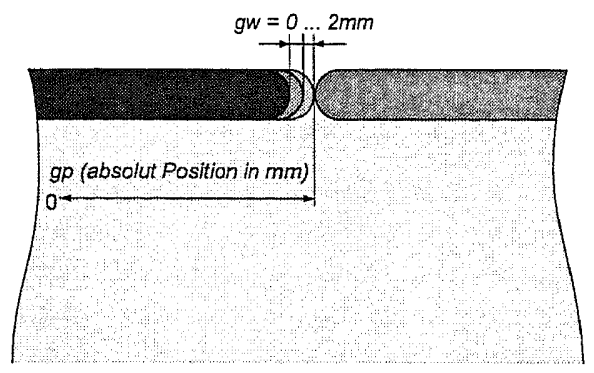

Figure 8. Schematic drawing of the FE model used to investigate the effect of different crack widths.

In order to know how the parameters have to be changed depending on the gap width, a simulation with one single gap at the surface was carried out (see figure 8). Then the width was parameterized in steps of $0.2 \mathrm{~mm}$ from 0 to $2 \mathrm{~mm}$ and the function of equation 6 was fitted to the different curves. Doing so the dependence of the parameters $y$ and $Y_{0}$ on the gap width could be found. The final form of equation 6 is:

$$
H_{y}(x, g w, g p)=\frac{-H_{g} \cdot L_{g}}{\pi}\left(\frac{(x-g p)}{(x-g p)^{2}+y(g w)^{2}}-\frac{(x-g p)}{(x-g p)^{2}+\left(Y_{0}(g w)+y(g w)\right)^{2}}\right)
$$

where, $g w$ is the gap width and $g p$ represents the gap position, while the parameters $H_{g}$ and $L_{g}$ were chosen to be constant. As it can be seen in figure 9, most of the disturbance can be removed by the subtraction of the two signals. In the frequency spectrum of the signals can be seen that especially the low frequency components could be removed from the original signal. The remaining noise can be removed easily by applying the same $13^{\text {th }}$ order low-pass filter as it was used in section 5.1 . 

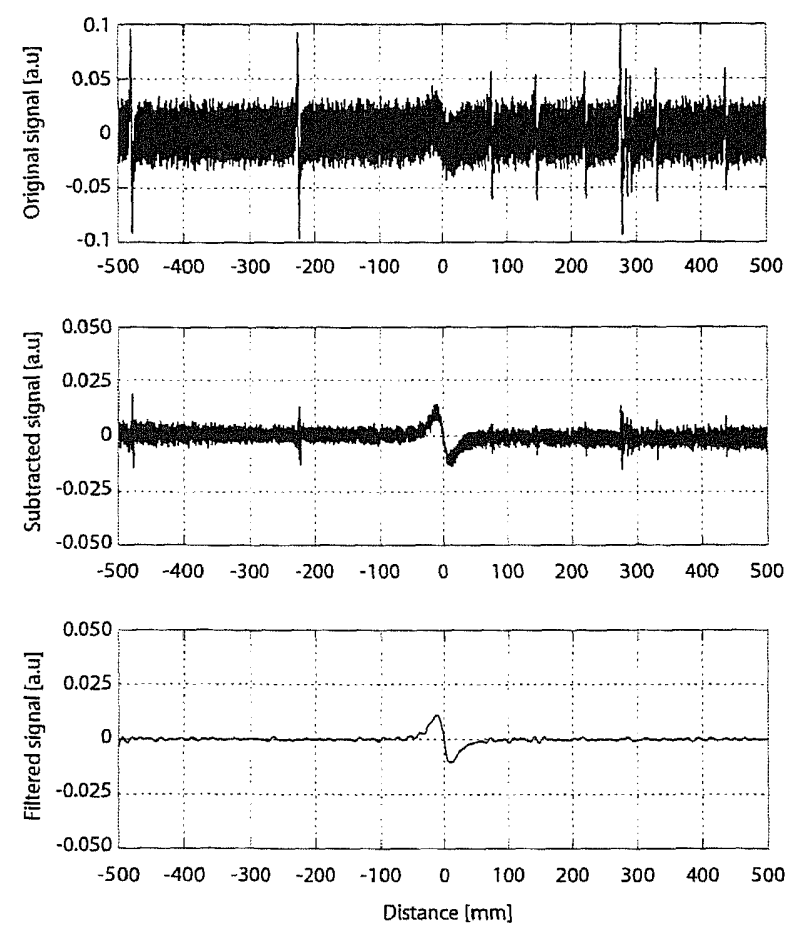

Figure 9. Application of the signal synthesis approach on calculation C5.

\section{CONCLUSIONS AND OUTLOOK}

A laboratory set-up with different damage scenarios, like wire breaks and corrosion, was built up. The measurements performed on this set-up showed, that it is possible to detect wire breaks as well as corrosion within an unwrapped steel cross-section. After the cable was wrapped, the signal is covered by the disturbance caused by the wrapping. Therefore, a finite element model was designed to investigate the disturbance. The influence of such a wrapping on the measured or calculated signals could be characterized under different conditions. Furthermore, possible filtering techniques were applied to the data and compared. Finally, an approach, based on a low-pass filter and an appropriate preprocessing of the signals is presented to filter the NDT signals obtained from wrapped steel cables. The signal synthesis described in section 5.3 is based on an analytical model of surface slots and requires the knowledge of the exact position of every wire. As shown in the last section, the principle could be applied to the data obtained from the FE model. Therefore, it is worth investing the time needed to overcome the practical problems, that have to be solved before this method can be applied to real cables. As seen, the exact determination of the relative position of single wires on the cable surface is essential for the success of the preconditioning. A possible solution to this problem are inductive proximity switches, or other short distance sensors which will be integrated in the measuring device. The signal of such sensors would have to be sampled with a high frequency in order to detect the position of each wire. Assuming a wire diameter of $2.5 \mathrm{~mm}$ and a velocity of $0.5 \mathrm{~m} / \mathrm{s}$ for the measuring device, 200 wires have to be detected in a second. But the detection of a wire is not enough, rather the position has to be determined as precisely as possible. Therefore, at least a few measuring points are required for each wire, which is given when a sampling frequency of $1 \mathrm{kHz}$ or more is used. In the next step, different approaches for the determination of wire positions will be implemented as prototypes, in order to find a satisfying method. The final verification of the method will be done on real measured data obtained from the cable model at Empa laboratory. 


\section{ACKNOWLEDGEMENT}

We thank Prof. Raimondo Betti of the Civil Engineering \& Engineering Mechanics Department of Columbia University, New York for making the corroded steel bundle, which was used in one of the experiments, available.

\section{REFERENCES}

1. A. Bergamini, "Nondestructive testing of stay cables - field experience in south east asia," in Third World Conference on Structural Control, F. Casciati, ed., 2, pp. 1057-1064, (Como), 2002.

2. R. Christen, A. Bergamini, and M. Motavalli, "Three-dimensional localization of defects in stay cables using magnetic flux leakage methods," Journal of Nondestructive Evaluation 22(3), pp. 93-101, 2003.

3. R. Christen and A. Bergamini, "Automatic flaw detection in nde signals using a panel of neural networks," $N d t \& E$ International 39(7), pp. 547-553, 2006. Christen, R. Bergamini, A.

4. P. A. Ivanov, Z. Zhang, C. H. Yeoh, L. Udpa, Y. Sun, S. S. Udpa, and W. Lord, "Magnetic flux leakage modeling for mechanical damage in transmission pipelines," leee Transactions on Magnetics 34(5), pp. 3020-3023, 1998. 2 Part 1.

5. R. Schifini and A. C. Bruno, "Experimental verification of a finite element model used in a magnetic flux leakage inverse problem," Journal of Physics D-Applied Physics 38(12), pp. 1875-1880, 2005. 31.

6. D. E. Bray, Nondestructive Evaluation, CRC Press, revised ed., 1997.

7. S. J. Orfanidis, Optimum Signal Processing: An Introduction, Prentice-Hall, Englewood Cliffs, NJ, 2nd ed., 1996. 